Ballester-Beltrán, J., Lebourg, M., Rico, P., and Salmeron-Sanchez, M. (2015) Cell migration within confined sandwich-like nanoenvironments. Nanomedicine, 10(5), pp. 815-828.

Copyright (C) 2015 Future Medicine Ltd.

A copy can be downloaded for personal non-commercial research or study, without prior permission or charge

Content must not be changed in any way or reproduced in any format or medium without the formal permission of the copyright holder(s)

When referring to this work, full bibliographic details must be given

http://eprints.gla.ac.uk/103606

Deposited on: 27 April 2015

Enlighten - Research publications by members of the University of Glasgow http://eprints.gla.ac.uk 


\section{Cell migration within confined sandwich-like nanoenvironments}

\section{Abstract}

4 Aim: We introduced sandwich-like cultures to provide cell migration studies with 5 representative nano-bio-environments where both ventral and dorsal cell receptors are

6 activated. Methods: We have investigated different nano-environmental conditions by 7 changing the protein coating (fibronectin, vitronectin) and/or materials (using polymers

8 that adsorb proteins in qualitatively different conformations) of this sandwich system to 9 show their specific role in cell migration. Results: Here we show that cell migration 10 within sandwich cultures greatly differs from 2D cultures and shares some similarities 11 with migration within 3D environments. Beyond differences in cell morphology and 12 migration, dorsal stimulation promotes cell remodeling of the ECM over simple ventral 13 receptor activation in traditional $2 \mathrm{D}$ cultures.

15 Keywords: sandwich culture, 3D cell migration, cell motility, lobopodia-based migration, fibronectin 
23 Cell migration is an essential process from embryonic development to adulthood.

Hence, studying cell migration on well-defined nano-bio-environments is required to better understand cellular mechanisms, search for novel therapeutic targets and design optimal implants. Mechanisms of cell migration have been extensively studied in vitro on two-dimensional (2D) surfaces, where it is described as a lamellipodia-based event consisting mainly of 4 steps: extension of the leading edge, adhesion formation, traction generation and subsequent retraction of the trailing edge [1]. So, cell migration on 2D substrates is integrin-mediated and depends on the traction force cells exerce on the underlying material. Nevertheless cell migration has been shown to differ between the traditional 2D models in vitro and the in vivo situations [2-5]. When switched to threedimensional (3D) culture systems - more representative of the in vivo environment different types of single cell migration can be described: (i) mesenchymal migration which is proteolytic dependent and lamellipodia-based - and thus similar to 2D migration (with implication of integrins and cell contractility) -; (ii) amoeboid migration which is non-proteolytic dependent and where adhesions are inexistent or very weak [68] and (iii) lobopodia-based migration which is determined by large cylindrical protrusions and depends on the 3D matrix elasticity [9]. Additionally, cells of the same type can switch between different modes of cell motility in response to the physical properties of the environment, integrin impairment, degradability and soluble signalling factors [9-12].

Given the difficulty of mimicking the in vivo nanoenvironment of the extracellular matrix (ECM), different complex 3D culture systems have been used to study cell 
migration [11, 13-15]. Unfortunately, differences inter- and even intra- 3D systems as well as difficulties in having reliable 2D controls hinder identifying and understanding the key features in 3D cell migration. Additional parameters play an important role in 3D migration such as (i) pore size [11], (ii) physical properties, e.g. matrix stiffness [9], (iii) nutrients diffusion [16], (iv) degradability of the system [17], (v) topographical cues [18], (vi) functionalities of the material (i.e. synthetic materials such as PEG hydrogels should be modified with adhesion domains in order to permit cell adhesion) [19] and (vii) variability of components from batch to batch [20]. Moreover, proper quantitative methods for analysis of migration rates in $3 \mathrm{D}$ environments are still lacking. New procedures to better understand cell migration within 3D cultures are therefore needed.

The distribution of cell receptors anchored to the ECM within 3D microenvironments highly differs from that observed on traditional 2D cultures. This is thought to be one of the key causes for the different cell behaviour between 2D and 3D systems since integrins trigger multiple signaling pathways which determine e.g. cell growth and gene expression [21-23]. Furthermore, differences in integrin localization and expression between $2 \mathrm{D}$ and $3 \mathrm{D}$ cultures have been described, supporting the hypothesis that different outside-in signaling may be the cause of different cell behavior [2, 24].

We have previously observed that sandwich-like microenvironments provide dorsal stimulation similar to 3D systems and trigger cell signaling pathways that promote a cell behavior more similar to 3D environments [25-27]. Here, we hypothesize that the excitation of dorsal receptors - by sandwiching cells already attached on a 2D surface - 
might switch cell migration towards 3D modes. To assess this hypothesis, we studied L929 fibroblast migration within sandwich-like cultures. Due to the high versatility of the system, different well-controlled nanoenvironments can be explored to investigate different parameters such as the protein conformation at the material interface. This technology then becomes an interesting tool to study some specific aspects of cell migration. In particular, we have varied the cell seeding procedure (culturing either isolated cells or as in wound healing assays), the chemistry of the ventral surface (using poly(ethyl acrylate), poly(methyl acrylate) and glass) and the biological input coming from the dorsal substrate (coating samples with different proteins, e.g. fibronectin (FN) and vitronectin (VN)) (see figure 1). Poly(lactic acid) (PLLA) was used as dorsal substrate in all cases. Cell morphology, adhesion and migration under different culture conditions were characterized, as well as the influence of the dorsal stimulation on the ability of cells to remodel the ECM.

\section{Materials and methods}

\section{Materials}

Polymer films of ethyl acrylate and methyl acrylate (EA and MA respectively, SigmaAldrich) were obtained by radical polymerization of a monomer solution using benzoin (98\% pure, Scharlau) as photoinitiator at $0,35 \mathrm{wt} \%$ and $1 \mathrm{wt} \%$ respectively. The polymerization was carried out up to limiting conversion. Spin-coating was then used to produce thin films of these polymers on glass coverslips (Brewer Science, Rolla, MO). Polymer solutions were made in toluene with $2.5 \%$ PEA or $6 \%$ PMA and spin-coating at $2000 \mathrm{rpm}$ for $30 \mathrm{~s}$. Finally, samples were dried at $60{ }^{\circ} \mathrm{C}$ in vacuum for $1 \mathrm{~h}$ before its use as ventral substrates. 
94 Thin films of PLLA were prepared by solvent casting a solution of $2 \%$ PLLA in 95 chloroform (Scharlau) in stainless steel washers and allowed to evaporate. Resulting

96 films were then thermally treated at $120^{\circ} \mathrm{C}$ for 5 minutes and used as dorsal substrates.

\section{Protein adsorption}

99

100

101

102

103

104

105

106

107

Fibronectin from human plasma (FN; Sigma) at $20 \mu \mathrm{g} / \mathrm{ml}$ in DPBS, vitronectin at 10 $\mu \mathrm{g} / \mathrm{ml}$ in DPBS (VN; Sigma) or heat-denatured Bovine Serum Albumin Fraction V (BSA; Roche, Barcelona, Spain) at $10 \mathrm{mg} / \mathrm{mL}$ in MilliQ water were adsorbed on the different substrates during $1 \mathrm{~h}$ at room temperature. After adsorption, samples were rinsed in DPBS to eliminate the non-adsorbed protein.

\section{Atomic Force Microscopy}

Atomic force microscopy (AFM) was performed on a JPK Nanowizard 3 BioScience AFM (JPK, Germany). Images were taken operating in the AC mode and analysed by the SPM and DP 4.2 software version. Si-cantilevers with a force constant of $2.8 \mathrm{~N} / \mathrm{m}$ and a resonance frequency of $75 \mathrm{kHz}$ (Nanoworld AG, Switzerland) were used. The phase signal was set to zero at a frequency $5-10 \%$ lower than the resonance one. Drive amplitude was $700 \mathrm{mV}$ and the amplitude set point was $700 \mathrm{mV}$.

\section{Cell culture}

L929 fibroblasts were maintained in Dulbecco's Modified Eagle Medium (DMEM) supplemented with $10 \%$ fetal bovine serum and $1 \%$ penicillin-streptomycin (Lonza). 
116 Prior to seeding, samples were sterilized under UV for $30 \mathrm{~min}$ (30 min each side in the 117 case of the dorsal substrates) and coated as described before. Then L929 cells were 118 seeded in DMEM without serum in order to direct specific fibronectin-cell adhesion. 119 After $3 \mathrm{~h}$ of culture, sandwich cultures (SW) were obtained by gently laying the dorsal 120 substrate over the seeded ventral substrate where cells were already adhered (figure 1). 121 In order to better mimic SW conditions, a washer without PLLA was laid on the 2D samples. Since several conditions have been studied, a specific nomenclature was used overall the study: $\mathrm{SW}_{x}^{y}$ with $x$-ventral and $y$-dorsal material condition. More detailed cell culture procedures can be found in the supplementary data.

\section{Time-lapse cell imaging}

Images were acquired every $20 \mathrm{~min}$ for $24 \mathrm{~h}$ using a Leica DMI 6000 inverted microscope (Leica Microsystems) with a 10X dry objective. During the observation, the samples were maintained at $37{ }^{\circ} \mathrm{C}$ and supplied with a $95 \%$ air and $5 \% \mathrm{CO}_{2}$ humidified gas mixture. Phase contrast images were gathered at 20 min intervals along $24 \mathrm{~h}$ of culture. For gap closure measurements, images were processed using the external plugin MiToBo in ImageJ software in order to obtain quantitative data about the dynamics in cell migration $[28,29]$.

\section{Immunofluorescence}

Samples were fixed in $10 \%$ formalin solution (Sigma) at $4{ }^{\circ} \mathrm{C}$ for $30 \mathrm{~min}$, "desandwiched" and then permeabilised for 5 minutes. After that samples were incubated in $1 \% \mathrm{BSA}$ in order to reduce the background signal. Cells were then incubated with the 
primary antibody (anti-vinculin and anti-paxilin antibodies (Sigma), anti-MMP2 and anti-MMP13 (Abcam), anti- $\alpha$-tubulin (Abcam), anti- $\beta_{1}$ integrin (BD Bioscience) or anti- $\alpha_{\mathrm{V}}$ integrin subunit (Millipore)) for 1 hour. Samples were then rinsed in DPBS/0.5\% Tween 20, followed by incubation with Cy3 or AlexaFluor 488 conjugated secondary antibody (Jackson Immunoresearch and Invitrogen respectively). If necessary, samples were incubated in BODIPY FL phallacidin (Molecular probes) diluted 1/100 in order to stain the cytoskeleton. Finally the samples were washed before being mounted in Vectashield containing 4',6-diamidino-2-phenylindole (DAPI, Atom).

\section{FN reorganization}

The ability of cells to reorganize the FN adsorbed on the ventral material surface was evaluated after $7 \mathrm{~h}$ of culture. Samples were fixed, "de-sandwiched" and subjected to FN immunodetection. The monoclonal primary antibody HFN7.1 that specifically binds

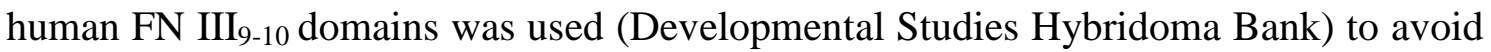
cross-reaction with FN secreted by murine cells.

\section{Image analysis}

156 Cell morphology was quantified by calculating different parameters using ImageJ software. Cell area, aspect ratio (major axis/minor axis), circularity (4 $\pi \quad \mathrm{x}$ 158 area/perimeter $\left.{ }^{2}\right)$ and roundness $\left(4 \times\right.$ area $\left./ \pi \times[\text { major axis }]^{2}\right)$ (the last 2 ones getting a 159 value of 1 for a perfect circle) were evaluated by analyzing at least 40 cells for each condition [30]. Additionally, cell morphology and distribution of focal adhesions were analyzed by The Focal Adhesion Analysis Server [31]. 


\section{Statistical analysis}

164 Results are shown as average \pm standard deviation. All experiments were performed at 165 least three times in triplicate. Results were analyzed by one-way ANOVA and if 166 significant differences were determined, Tukey's post-hoc test was performed. For each 167 sandwich condition, the bottom substrate as 2D culture was used as its specific control. 168 Statistically significant differences are indicated with $* \mathrm{P}<0.05$ and $* * * \mathrm{P}<0.001$.

\section{Results and discussion}

\section{Phenomenology of cell migration within sandwich environments}

172 We have studied L929 fibroblasts migration within sandwich cultures using a modified 173 wound healing assay based on culture inserts since manual scratching stripped off the 174 thin spin-coated polymer films (figure 1). Moreover manual scratching of cell 175 monolayers has been correlated to changes in cell morphology, alteration of 176 proliferation and deregulation of migration [32]. 
A

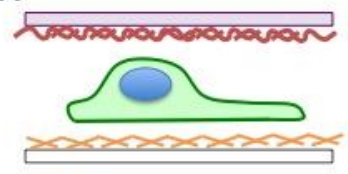

Dorsal substrate: PLLA film

m Dorsal protein: FN, VN, BSA, Ø

* Ventral protein: FN

$\square$ Ventral substrate: glass, PEA, PMA

C

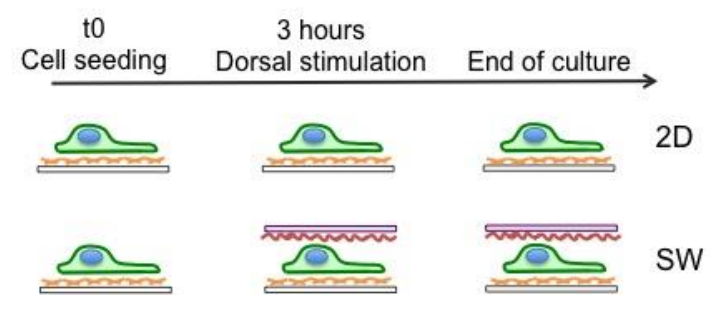

B Wound healing

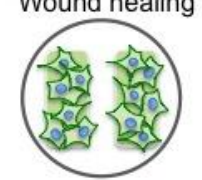

Isolated cells

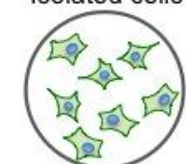

Figure 1. Summary of the sandwich-like culture conditions. (A) Sketch of the sandwich-

like model and the different combinations of material and proteins. (B) Sketch of the two different cell cultures used during this work. (C) Timing diagram showing the procedure followed for sandwich culture systems.

Movie 1 (supplementary material) shows cell migration on FN coated glass coverslips (2D) and then after overlying with a FN-coated PLLA film (sandwich culture, SW).

Cells on the $2 \mathrm{D}$ control migrate according to the classical 4 migration steps, by adopting a polygonal shape with wide lamellipodia and pseudopodia projections [33, 34]. However, cells within sandwich culture adopted an elongated morphology with fewer (usually 1 or 2 ) but longer pseudopodia that were more persistent in time resembling what has been observed in other 3D environments (figure 2A) [9, 35, 36]. Such elongated cell morphology of migrating cells has not been previously observed within our sandwich microenvironments [25-27]. Importantly, also note that the nuclei of cells migrating in sandwich environments hardly move from their initial position, which suggests that the last steps of the migration process (traction generation and the retraction of the trailing edge) were hindered or not coordinated [37]. 
195 One might think that the elongated morphology observed within the sandwich culture at 196 the front edge of the 2 cell populations in a wound healing assay might be related to the 197 biochemical crosstalk between the 2 populations (chemotaxis due to increased concentration of cell secreted signals within the SW environment), which may enhance extension directionality and persistence. However these elongated cells were observed along the whole border of the seeded fields, not only the ones facing the wound. So we first assessed whether this elongated morphology was due to the high cell density used during the wound healing-like assay rather than to the specific sandwich condition. Hence, isolated cells were cultured at low density and migration was monitored. Figure 2 depicts the morphology of selected cells after different time-points of culture so cell morphology and migration can be evaluated. Cells overlaid with the FN-coated dorsal substrate (sandwich culture, referred to as $\mathrm{SW}^{\mathrm{FN}}$ ) did not show such elongated morphology. Hence the cause of this elongated morphology should be sought as a consequence of combined dorsal stimuli and high cell density population, which suggests an important role of cell-cell contact and Rac1 signaling [38]. To corroborate this, high cell density areas of the wound healing were imaged and it was seen that only cells in the perifery of the population projected the long pseudopodia whilst cells inside the population conserved a polygonal shape (data not shown).

214 When the morphology of migrating cells within the sandwich culture was studied more in depth (figure 2) we saw that the circularity of isolated cells was similar to the

216 circularity of cells during the wound healing assay (figure 2B) and, most importlantly,

217 isolated cells within sandwich culture showed different roundness than isolated cells 
218 cultured on 2D substrates. These suggest that, despite not having such long

219 pseudopodia, cells sense the dorsal stimuli and respond to it by changing their

220 morphology as reported for other 3D systems [39, 40]. Moreover, we have recently

221 shown that cell morphology within sandwich culture highly depends on the substrate

222 properties similarly as happens in 3D cultures [27]. Regarding cell motility, cells

223 cultured on 2D substrates moved around the starting point in random open trajectories,

224 whereas cells within $\mathrm{SW}^{\mathrm{FN}}$ remain mainly static at the initial location, and only

225 cytoplasmatic extensions were projected (figure 2C). So, sandwich culture hinders cell

226 migration and nuclei movement both for isolated and wound healing cultures though

227 cells do project long pseudopodia only in the latter case.

229 In order to investigate the role of the nature of the dorsal stimulus, cells were studied

230 also when sandwiched with an un-coated dorsal substrate $\left(\mathrm{SW}^{\varnothing}\right)$. As a consequence

231 these cells received similar biological signals than cells on 2D samples, as the only

232 biologically driven interaction comes from the ventral substrate. As shown in figure 2C,

233 cells within $\mathrm{SW}^{\emptyset}$ can move and change their location, though less than on 2D

234 substrates. The lack of protein coating on the dorsal substrate is therefore related to an

235 increased cell migration compared to $\mathrm{SW}^{\mathrm{FN}}$, though cells do not behave as on the 2D

236 control. This suggests that not only the biological but also the mechanical stimuli are

237 important cues in sandwich environments [25-27]. 

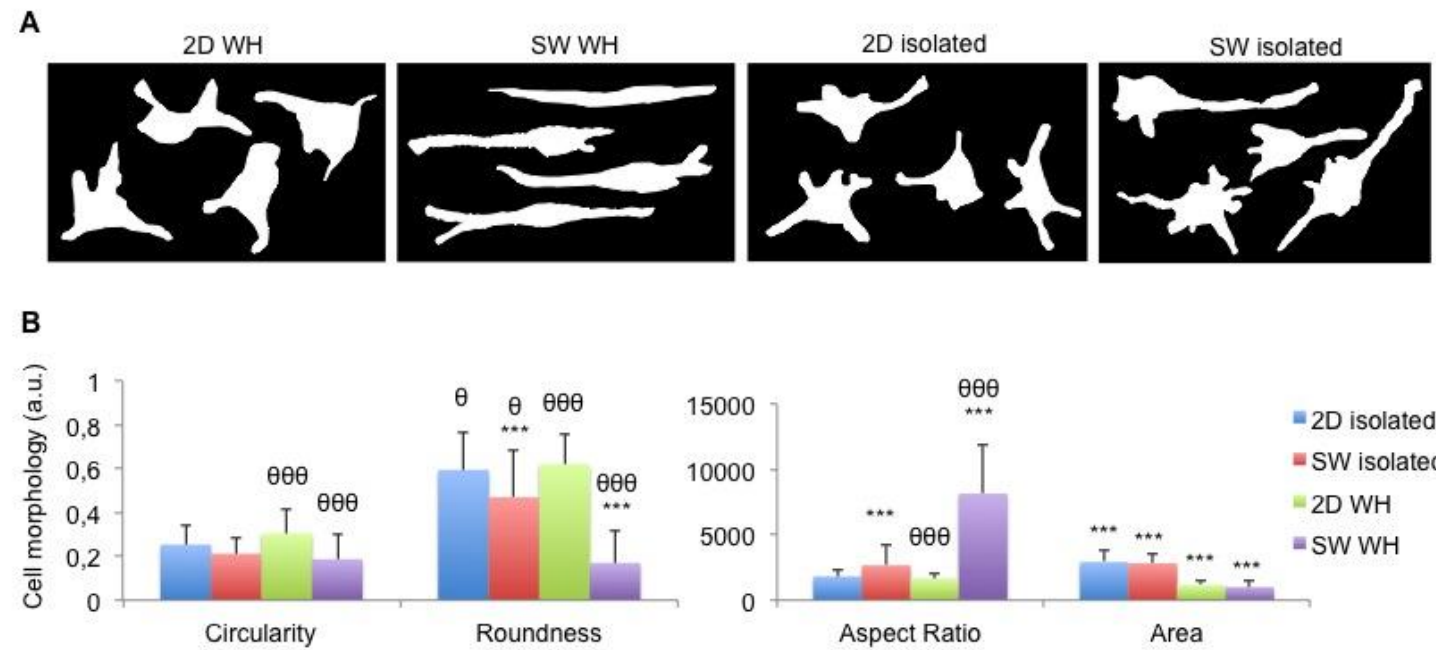

C

238
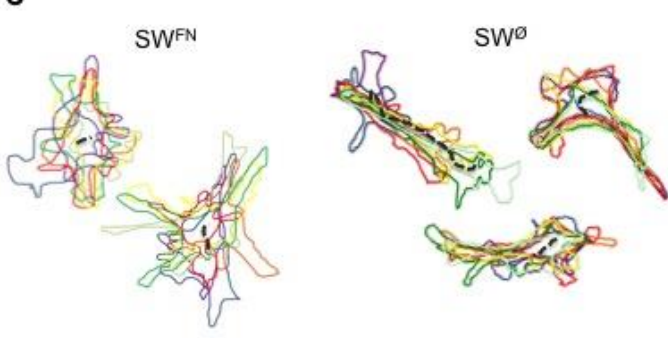
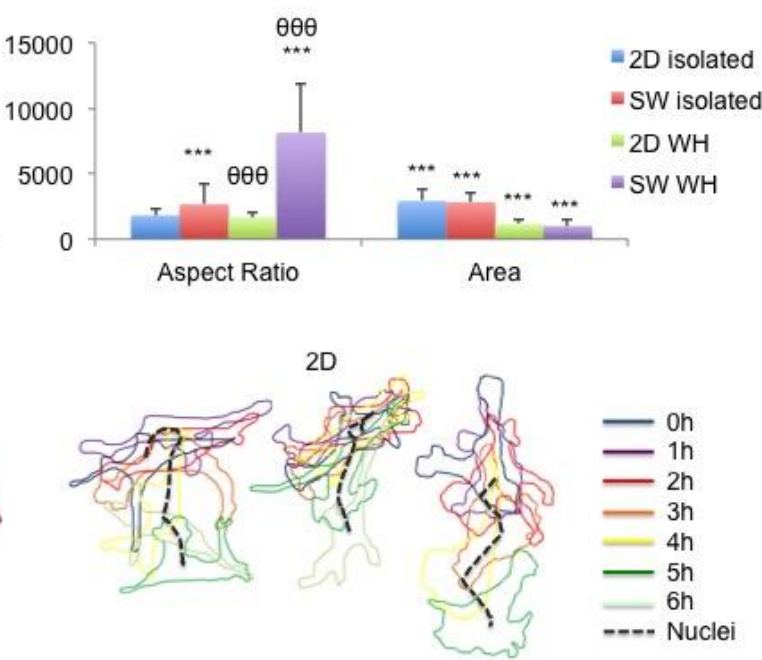

Figure 2. Cell migration and morphology. (A) Cell morphology after 12 hours of migration in a wound healing assay (WH) or as isolated cells (isolated).

Morphology of cells after 12 hours of culture. * shows significance comparing isolated with wound healing for the same type of dimensionality (2D or SW); and $\theta$ comparing 2D with SW for the same type of culture condition (isolated or wound healing). (C) Outlined cells after different time points of culture. Dotted lines represent the movement of cell nuclei. Coordinates are mantained for each outlined cell so that the displacement displayed in the picture is equivalent to the migration oberved during the culture.

\section{Characterization of the migrating cells}

Cells migrating in the wound healing assay were further characterized seeking to understand differences with $2 \mathrm{D}$ conditions. Cultures were kept for $7 \mathrm{~h}$ to allow cells to project these characteristic long pseudopodia within the sandwich culture. First, focal adhesion proteins and integrins were evaluated as it is well accepted that these differ 
between $2 \mathrm{D}$ and 3D/in vivo environments [2, 40-42]. Focal adhesion proteins such as

$254 \mathrm{pFAK}^{\mathrm{Ty} 925}$ and vinculin were detected at the cell edge when cultured on 2D substrates

255 (figure 3). Within sandwich microenvironments, these proteins were localized mainly at

256 the rear part of the cell and at the tip of the long pseudopodia. Besides, colocalization

257 between vinculin and $\mathrm{pFAK}^{\mathrm{Tyr} 925}$ was observed in both conditions. Further differences

258 between vinculin and pFAK did not only occur between both 2D and SW cultures but

259 also when comparing the leading and rear part of cells migrating within the sandwich

260 (table 1 and 2). Cells within the sandwich culture have a higher number of focal

261 adhesions at the rear part than at the leading edge (table 1). This might explain why

262 cells cannot retract the trailing edge during migration and only long pseudopodia are

263 projected. Likewise, the larger focal adhesions observed for the sandwich culture (as

264 well as the regulation of their turnover) might explain the lower migration rate $[37,43]$.

Table 1. pFAK and vinculin characterization in the leading and rear part of cells migrating within sandwich culture in a wound healing assay.

Leading

Rear

$P$ value

\begin{tabular}{l|lll|}
\hline \multicolumn{1}{c|}{$\boldsymbol{p F A K}$} & & \\
\# Focal adhesions & $165.11 \pm 67.33$ & $263 \pm 90.8$ & $<0.0001^{*}$ \\
Area $\left(\mu m^{2}\right)$ & $1.06 \pm 0.23$ & $0.81 \pm 0.18$ & $<0.0001^{*}$ \\
Total Area $\left(\mu m^{2}\right)$ & $164.47 \pm 41.92$ & $205.80 \pm 58.07$ & $0.0065^{*}$ \\
Distance to edge $(\mu m)$ & $1.83 \pm 0.52$ & $4.49 \pm 1.01$ & $<0.001^{*}$ \\
Major axis $(\mu m)$ & $1.46 \pm 0.2$ & $1.29 \pm 0.15$ & $0.0019^{*}$ \\
\cline { 2 - 4 }
\end{tabular}


Minor axis $(\mu m)$

$0.79 \pm 0.09$

$0.68 \pm 0.06$

$<0.0001$

\section{Vinculin}

\# Focal adhesions

Area $\left(\mu m^{2}\right)$

Total Area $\left(\mu m^{2}\right)$

Major axis $(\mu m)$

Minor axis $(\mu m)$

$\begin{array}{|llc|}163.27 \pm 68.83 & 279.56 \pm 106.67 & 0.0012 \text { * } \\ 0.84 \pm 0.31 & 0.88 \pm 0.25 & 0.68 \\ 133.56 \pm 65.62 & 234.45 \pm 71.45 & 0.0003^{*} \\ 1.36 \pm 0.28 & 1.41 \pm 0.21 & 0.54 \\ 0.67 \pm 0.11 & 0.71 \pm 0.10 & 0.30\end{array} \mid$

Table 2. pFAK and vinculin adhesions in 2D and sandwich cultures.

2D

SW

$P$ value

\begin{tabular}{l|lll|}
\hline \multicolumn{1}{c|}{$\boldsymbol{p} \boldsymbol{F} \boldsymbol{K} \boldsymbol{K}$} \\
\# Focal adhesions & $682.22 \pm 193.64$ & $382.75 \pm 98.31$ & $0.0014^{*}$ \\
Area $\left(\mu m^{2}\right)$ & $0.57 \pm 0.05$ & $0.69 \pm 0.14$ & $0.0014^{*}$ \\
Total Area $\left(\mu m^{2}\right)$ & $387.31 \pm 100.69$ & $258.33 \pm 64.95$ & $0.0046^{*}$ \\
Distance to edge $(\mu m)$ & $6.39 \pm 1.56$ & $3.56 \pm 0.89$ & $0.0003^{*}$ \\
Major axis $(\mu m)$ & $1.03 \pm 0.03$ & $1.20 \pm 0.14$ & $<0.0001^{*}$ \\
Minor axis $(\mu m)$ & $0.61 \pm 0.02$ & $0.65 \pm 0.06$ & $0.0154^{*}$
\end{tabular}




\begin{tabular}{l|llc|}
\hline \multicolumn{1}{c|}{ Vinculin } & & \\
\# Focal adhesions & $495.44 \pm 185.9$ & $278 \pm 114.29$ & $0.0197^{*}$ \\
Area $\left(\mu m^{2}\right)$ & $0.74 \pm 0.10$ & $0.66 \pm 0.09$ & 0.59 \\
Total Area $\left(\mu m^{2}\right)$ & $357.04 \pm 102.95$ & $240.05 \pm 50.17$ & $0.0108^{*}$ \\
Major axis $(\mu m)$ & $1.30 \pm 0.11$ & $1.33 \pm 0.17$ & 0.27 \\
Minor axis $(\mu m)$ & $0.61 \pm 0.03$ & $0.64 \pm 0.38$ & 0.07
\end{tabular}

279
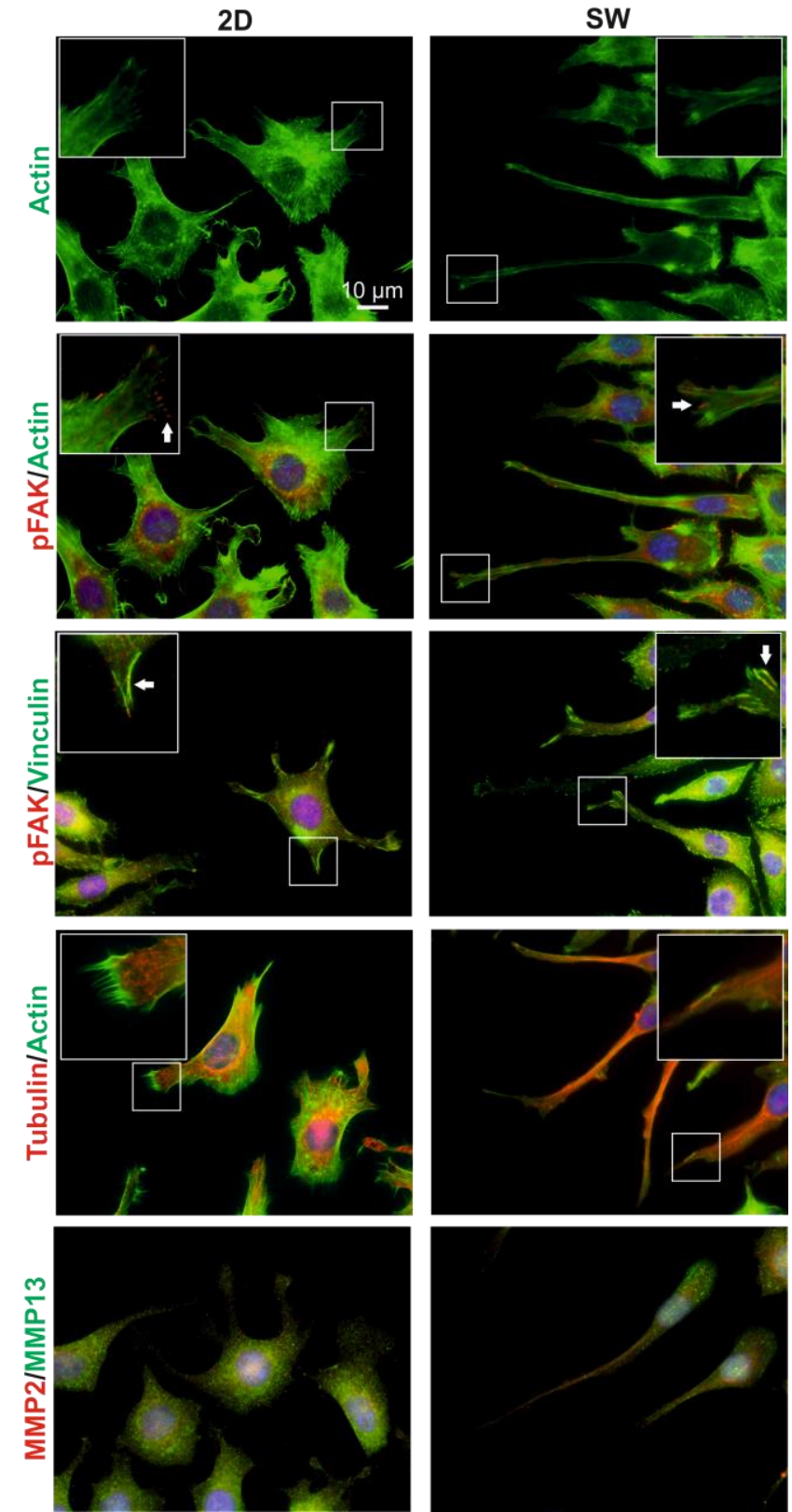
281 Figure 3. Immunofluorescence images after 7 hours of culture (2D and SW). Insets

282 show magnifications of focal adhesions and cell cytoskeleton. Arrows point out focal adhesions.

Stressed actin fibers and developed $\alpha$-tubulin microtubules were assembled both on 2D and in SW cultures, but with different cell morphology that confined nuclei into a narrower space within SW cultures. Likewise, broad actin lamellipodia were seen for cells seeded on 2D substrates but not for cells within the sandwich culture (figure 3 ). Similar elongated fibroblast morphology has been previously described for other 3D systems. In particular, elongated cells with multiple long protrusions and small lamellipodia at their tips (that contain Rac1, cortactin, PIP3 and Cdc42) have been shown to use lamellipodia-based migration, whereas elongated cells with fewer protrusions and no lamellipodia use the lobopodia-based migration. Interestingly, cells switch between these 2 migration modes according to the elastic properties of the 3D environment, which involve RhoA-ROCK-myosin II signalling [9]. Here we observe elongated cells in SW with less lamellipodia than cells on 2D and without lateral blebs (movie 1, figure 2 and figure 4), which corresponds to migration in a lamellipodia-based mode. However, cells display only 1-2 protrusions at the leading edge, which might suggest a new mode of migration mode for cells within SW environments intermediate inbetween lamellipodia and lobopodia-based migration (figure 4). This is likely a consequence of the environment provided by the sandwich system. Likewise, changing the inputs coming from the sandwich environment could lead to different cell migration (as in 3D systems [9]) similarly as it triggers differences in cell morphology, adhesion and differentiation [25-27, 39]. 
306 ECM degradation is another key step for 3D cell migration [11,9], as cells must

307 literally open spaces through the fibrillar mesh of proteins that constitute their

308 environment. We have investigated the expression of matrix metalloproteinases

309 (MMPs) - the main enzymes secreted by cells to degrade the ECM - and focused on

310 MMP2 and MMP13, that are known to degrade FN [44, 45]. However, no differences

311 were found for MMP2 and MMP13 on 2D and SW cultures (figure 3).

2D lamellipodia-based migration

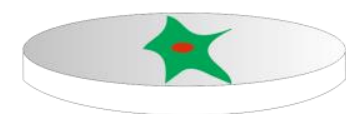

3D amoeboid migration

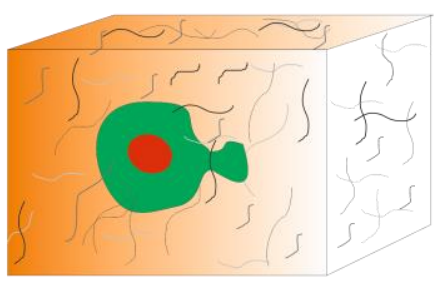

- Rounded cells with constricting ring

- Proteolysis independent
3D lamellipodia-based migration

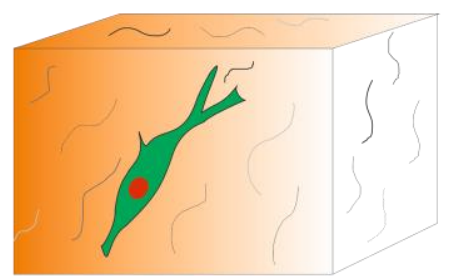

- Elongated cells with multiple long protrusions enriched with Rac1, cdc42 and PIP3

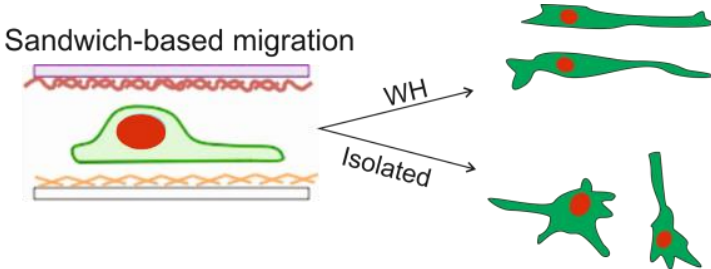

3D lobopodia-based migration

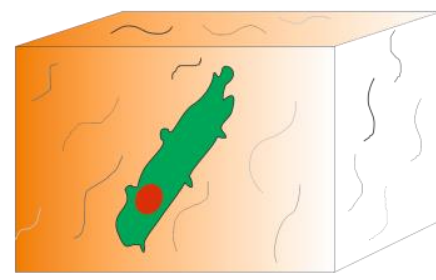

- Elongated cells with few front blunt cylindral protrusions and lateral blebs

- Non-polarized Rac1 and cdc42

312

Figure 4. Schematic representation of cell migration modes in 2D, 3D and sandwich culture. Differences in the migration mode are due to dimensionality, cell lineage and ECM properties.

\section{Ventral ECM remodeling during cell migration}

318 It has been found that fibroblasts migrating in the lamellipodia mode remodel their matrix but those in the lobopodia mode do not [9]. Hence, we investigated the role of 
320 SW environments in ECM remodeling. The fate of ventral FN was studied on FN-

321 coated glass (2D) overlaid with a dorsal FN-coated PLLA film (SW). As a positive

322 control cells were cultured with growth medium (10\% FBS) as this triggers ECM

323 reorganization [46]. It is important to remark here that for the rest of conditions cells

324 were cultured in serum-free medium, so that the sole influence of the sandwich

325 enviroment on cell reorganization was considered. Figure 5 shows that, regardless the

326 migration model used (wound healing or isolated cells), FN was not remodeled on 2D

327 substrates whilst cells formed new FN fibrils within sandwich cultures. Dorsal stimuli

328 did therefore trigger this ECM remodeling, as reported for FN and collagen in 3D

329 matrices $[9,47]$. In order to better understand this phenomenon, we cultured cells in SW

330 under different dorsal conditions by coating with different proteins (vitronectin, bovine

331 serum albumin and un-coated; $\mathrm{SW}^{\mathrm{VN}}, \mathrm{SW}^{\mathrm{BSA}}$ and $\mathrm{SW}^{\varnothing}$ respectively). As controls we

332 used 2D samples where the corresponding dorsal protein was included in the culture

333 medium, so that dorsal receptors were biologically but not mechanically stimulated. As

334 summarised in table 3, FN reorganization only occurred in sandwich cultures regardless

335 of the protein coating used, even occurring when the dorsal PLLA was coated with BSA

$336-\mathrm{SW}^{\mathrm{BSA}}$ (a non-adhesive protein) - or left uncoated $\left(\mathrm{SW}^{\varnothing}\right)$. Hence the mechanical dorsal

337 input has a key role to trigger FN reorganization and determine cell fate (figure 5 and 338 table 3). 

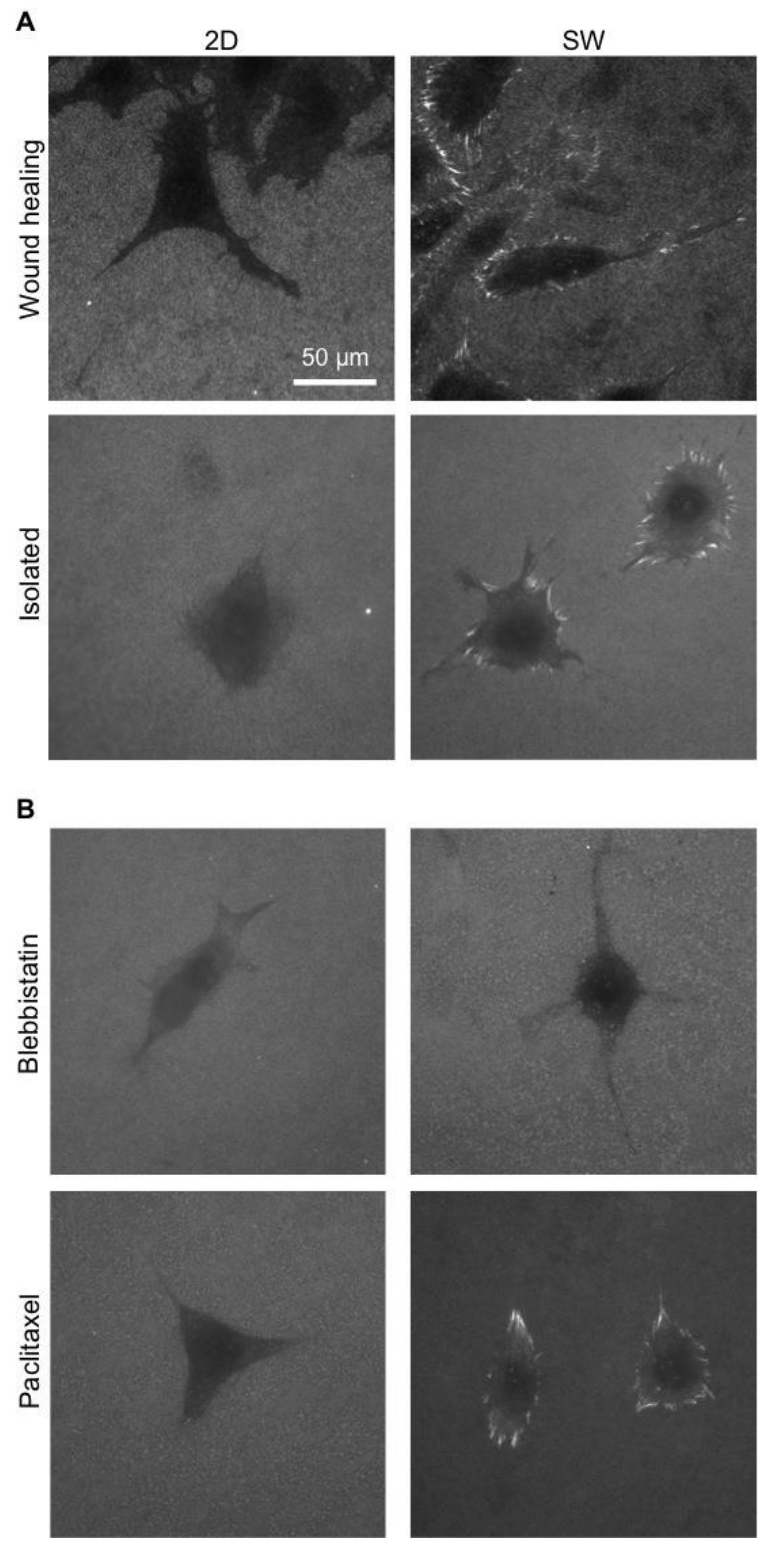

341 Figure 5. Ventral FN reorganization. (A) Sandwich culture triggers ventral FN reorganization by forming new fibrils both in isolated and wound healing cultures. (B)

343 Cell contractility is needed to reorganize FN within the sandwich culture.

345 Cell contractility is known to influence cell migration $[9,48,49]$ via phosphorylation of 346 myosin light chain (MLC) so we examined whether this might also be related to the 347 ECM reorganization process triggered by SW environments. To do so cells were 348 cultured in the presence of pharmacological inhibitors that impair contractility such as 
349 the Rho/ROCK pathway inhibitor Y-27632 and Blebbistatin, a specific inhibitor of 350 myosin II activity [50, 51]. In addition, cells were also cultured in the presence of 351 Paclitaxel that stabilizes microtubules thus showing an opposite effect [52]. 352 Contractility inhibitors (Y-27632 and Blebbistatin) impaired the ventral FN 353 reorganization within sandwich cultures whilst Paclitaxel enhanced it (figure 5B and 354 table 3). Cell contractility is therefore needed to reorganize FN within the sandwich 355 culture. Sandwich culture might therefore enhance cell contractility to enable the 356 reorganization of ventral $\mathrm{FN}$.

Table 3. Summary of the ventral FN reorganization. + stands for reorganization, - for no reorganization and $*$ for the halo seen on PEA (see next section).

\section{D SW}

Growth medium $(+\mathrm{C})$

Medium w/o serum

Blebbistatin/Y-27632

Paclitaxel

$\mathrm{SW}^{\mathrm{FN} / \mathrm{VN} / \mathrm{BSA} / \varnothing}$

FN/VN/BSA dissolved in medium

Ventral PEA/PMA

\begin{tabular}{|c|c|}
+ & + \\
- & + \\
- & - \\
- & + \\
- & + \\
$-*$ & -
\end{tabular}

360 


\section{Role of the ventral substrate in cell migration}

365

366

367

368

369

370

371

372

373

374

375

376

377

378

379

380

381

382

383

384

385

386

387

Next, we wanted to address if the elongated morphology and migration rates found for migrating cells within SW environments might be tuned by using different materials as ventral substrates. We used: (i) FN-coated glass, where FN adsorbs loosely in a globular conformation that can be easily reorganized by cells [53], (ii) spin-coated poly(ethyl acrylate) (PEA) on which FN assembles spontaneously into fibrillar (nano)networks $[54,55]$ and (iii) spin-coated poly(methyl acrylate) (PMA) on which FN adopts a globular conformation (figure 6) [56]. Figure 6A shows the cellular reorganization of adsorbed FN after $5 \mathrm{~h}$ of culture (in growth media) on the different $2 \mathrm{D}$ substrates (chemical structures shown in figure 6B). As expected, new FN fibrils were only formed on glass and PLLA (white arrows) as cells are unable to reorganize FN on PEA nor PMA due to the strength of the protein-material interactions [57]. Additionally we addressed whether the ECM reorganization triggered by the dorsal stimuli in SW cultures (using glass as ventral substrate) was also observed on PEA and PMA in the absence of serum. Cells did not reorganize ventral FN on these surfaces, which suggests that the dorsal stimulus is not strong enough to overcome the protein-material interaction (table 3). Besides, FN showed a halo around cells when cultured on 2D PEA substrates. This phenomenon has been further investigated and correlated with the strength of FN-PEA interactions [58].

After assessing that removal of the culture insert did not alter the characteristic FN adsorption and distribution on PEA and PMA (network and globular-like conformation respectively; figure 6D), cell migration on the different materials was followed using the wound healing assay. Gap closure, defined as the percentage of the initially void 
surface that is colonized by cells, is shown in figure 6E. Polygonal cells were observed on every $2 \mathrm{D}$ substrate whilst cells adopted an elongated morphology when cultured under sandwich culture regardless of the material used. On the other hand, differences in cell migration were clearly observed between 2D and SW cultures for the same ventral substrate. Cells migrated longer distances on $2 \mathrm{D}$ substrates than within the SW environment, which increased gap closure (figure 6E). Finally, no differences in gap closure were oberved for 2D samples but, strikingly, significant differences were observed for the different SW cultures: gap closure occurred more efficiently using PMA than glass or PEA, which suggests that FN conformation (globular on PMA and fibrillar structures on PEA) and the strength of FN interaction with the material surface play a key role in cell migration (figure 6E). Note that these differences appear only within SW environments but not for the 2D control, which stresses the role of 400 dimensionality in cell migration.
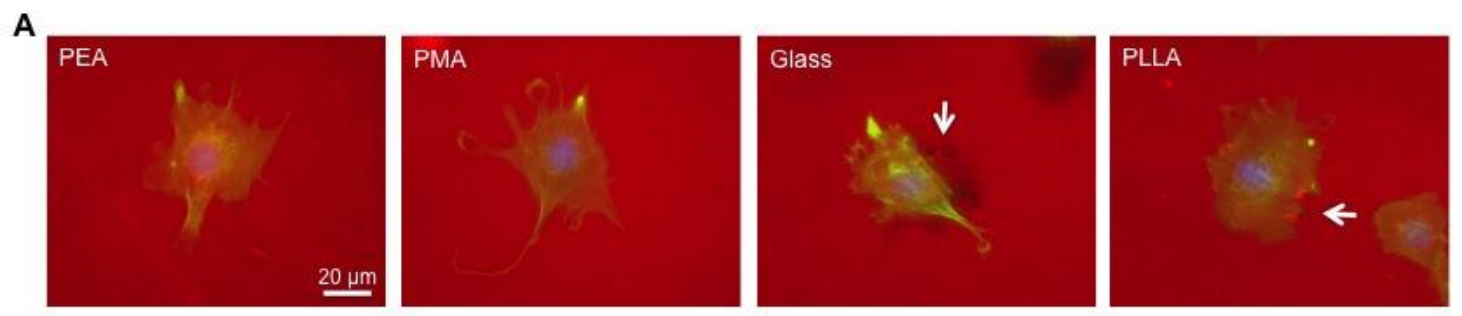

C

402

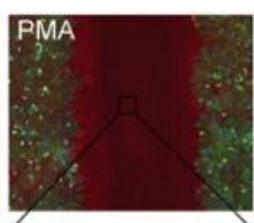

D
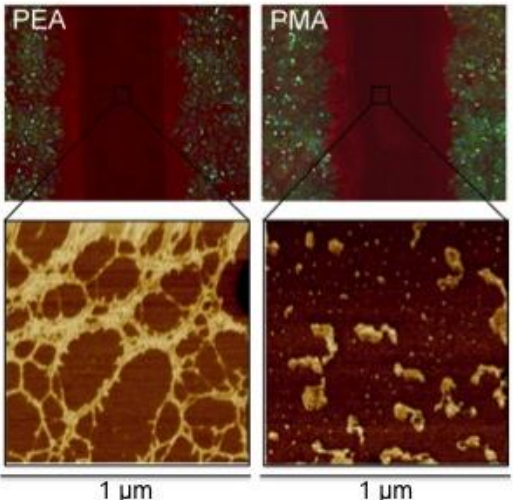

B<smiles>CCOC(=O)C(CC(C)C)C(C)C</smiles>

poly(ethyl acrylate), PEA

E

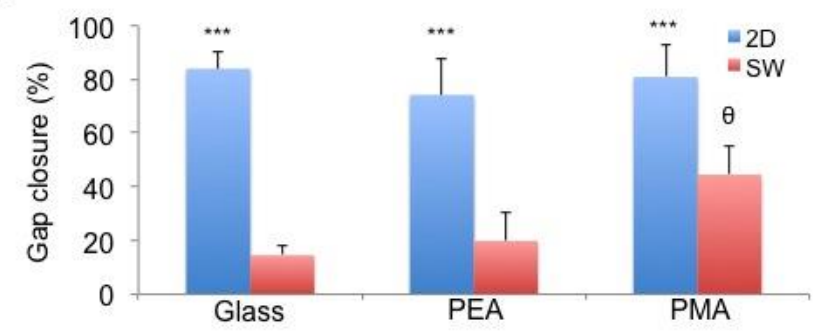


403 Figure 6. Cell migration using different ventral FN-coated surfaces. (A) Fibronectin 404 (red) is reorganized on 2D glass and PLLA but not on PEA and PMA. Actin 405 cytoskeleton is shown in green and nuclei in blue. White arrows show the formation of

406

407

408

409

410

411

412

413

414

415

416

417

418

419

420

421

422

423

424

425 newly cell-reorganized FN fibrils. (B) Chemical structure of PEA and PMA. (C) Fluorescence images show the FN-coated gap $(500 \mu \mathrm{m})$ between the two cell populations after removing the insert used for the wound healing assay. Actin cytoskeleton (green), nuclei (blue) and FN (red) are shown. (D) AFM images of FN after insert dettachment to confirm that $\mathrm{FN}$ distribution is not altered on the migrating area. (E) Gap closure after $24 \mathrm{~h}$ of wound healing assays using glass, PEA and PMA as ventral substrates. $*$ shows significance comparing conditions with similar ventral substrate; and $\theta$ when comparing results of the same dimensionality (2D or SW).

\section{Role of dorsal stimuli in cell migration}

We next examined the role of dorsal stimuli in cell migration. To do so we used FN coated glass as ventral substrate and as dorsal substrate (i) PLLA coated with different proteins ( $\mathrm{FN}$ or $\mathrm{VN}$ ) to address the role of integrin-mediated interactions throughout different proteins and (ii) bare surfaces $(\varnothing)$ to address the effect of pure mechanical stimuli $\left(\mathrm{SW}^{\varnothing}\right.$ might not induce any dorsal biological interaction but a response due to the bare surface).

As observed previously, cells adopted polygonal morphology on 2D substrates and elongated ones within sandwiched cultures. No differences in either gap closure or cell 
morphology were observed regardless of the use of dorsal FN or VN coatings.

427 Strikingly, when overlaid by a non-adhesive dorsal substrate $\left(\mathrm{SW}^{\varnothing}\right)$, cells maintained 428 the characteristic elongated morphology found in SW cultures but migrated more

429

430

431

432

433

434

435

436

437

effectively (figure 7 and movie 2). So, even when the SW provided a pure mechanical input (no protein coating), cell migration greatly differed. This observation, together with results included in figure 2 and previous works [25], suggests an important role for the dorsal mechanical input in cell behavior. Hence, we hypothesize that cell migration is increased under this condition ( $\mathrm{SW}^{\emptyset}$; both for isolated cells and wound healing assay) compared with $\mathrm{SW}^{\mathrm{FN}}$ and $\mathrm{SW}^{\mathrm{VN}}$ due to the interactions with the extra (dorsal) layer of proteins in the latter cases, and the regulation of focal adhesion formation and turnover on both dorsal and ventral substrates.

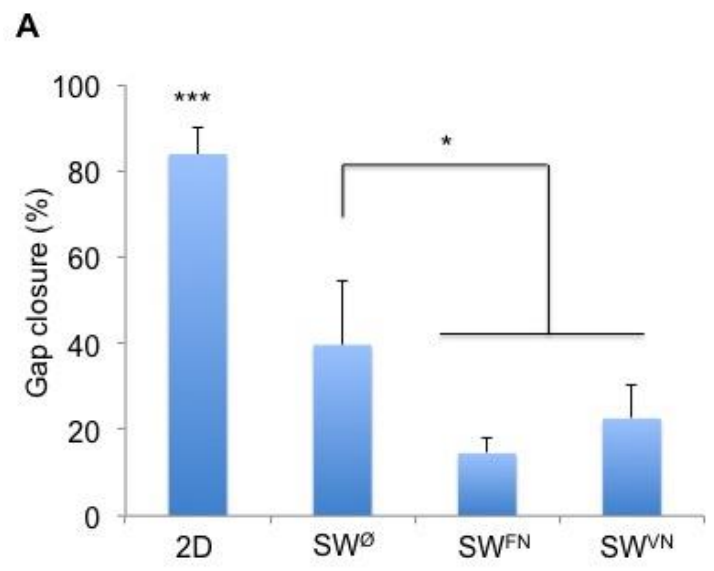

B
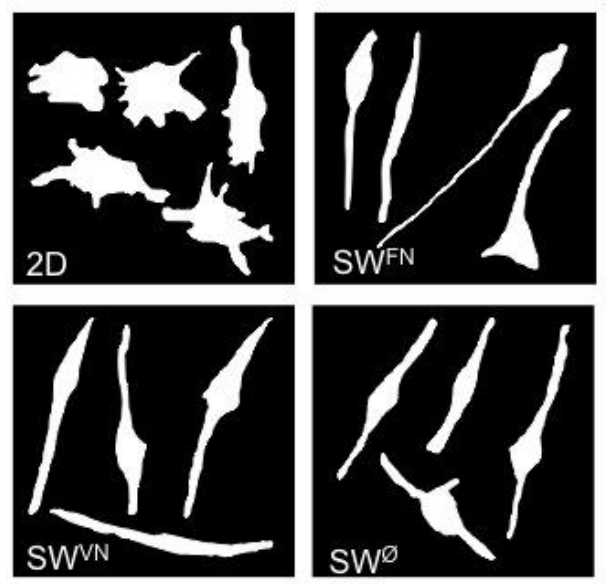
439 Figure 7. Wound healing assay under different dorsal stimuli (2D and SW cultures) (A)

440 Migration rates after 24 hours of culture using FN-coated glass as ventral substrate and

441 bare PLLA $\left(\mathrm{SW}^{\emptyset}\right)$ or coated with either $\mathrm{FN}$ or $\mathrm{VN}\left(\mathrm{SW}^{\mathrm{FN}}\right.$ and $\mathrm{SW}^{\mathrm{VN}}$ respetively) as

442 dorsal substrate. (B) Representative cell morphology of migrating cells for each 443 condition.

\section{Executive summary}

Background

- Cell migration is an essential process in vivo

- Robust culture systems mimicking the in vivo environment are needed

Results

- Sandwich culture offers a closer 3D environment and is a versatile system to sandwich environments

\section{Future perspective}

Cell migration is an essential process in many physiological and pathological 459 conditions. Providing novel tools to understand this process is therefore essential. Thus, 460 future work should develop more powerful and biomimetic systems and technologies to investigate this process in in vivo-like conditions. 
463 We have shown sandwich culture as a tool to investigate cell migration in a closer 3D

464 environment than the traditional 2D substrates. The versatility of this system allows the 465 study of cell fate under a wide spectrum of well-controlled conditions to better 466 understand cell behavior within 3D cultures such as stacked layers of cells or hydrogels.

467 Overall, cell morphology was highly influenced by the type of culture whereas cell 468 migration was determined by the inputs coming from both the ventral and dorsal 469 substrate. Furthermore, FN was reorganized in new fibrils when cells were dorsally 470 stimulated within the sandwich culture, showing that the dorsal excitation triggers 471 different signaling compared to 2D conditions. Our results suggest that both biological 472 and mechanical stimuli play an important role in cell migration. 
4821 Ridley AJ, Schwartz MA, Burridge K et al. Cell migration: integrating signals from 483 front to back. Science. 302(5651), 1704-1709 (2003).

4842 Cukierman E, Pankov R, Stevens DR, Yamada KM. Taking cell-matrix adhesions to 485 the third dimension. Science. 294(5547), 1708-12 (2001). differences in leukemia therapy. Cell Cycle. 10(10), 1540-1544 (2011). leukocytic uptake of oligodeoxynucleotides. Cell Mol Life Sci. 65(7-8), 1237-1247 491 (2008). Development. 141(10), 1999-2013 (2014). contact guidance: T-lymphocyte crawling through fibrillar collagen is independent of matrix remodeling by MMPs and other proteases. Blood. 102(9), 3262-3269 (2003). Opin Cell Biol. 16(1), 14-23 (2004). mammary tumors correlated with gene expression patterns revealed by molecular 501 profiling. Cancer Res. 62(21), 6278-6288 (2002). distinct modes of 3D cell migration. J Cell Biol. 197(3), 439-55 (2012). 
11 Wolf K, Mazo I, Leung H et al. Compensation mechanism in tumor cell migration: 509 mesenchymal-amoeboid transition after blocking of pericellular proteolysis. J Cell Biol. $510160(2), 267-277(2003)$.

51112 Friedl P, Wolf K. Plasticity of cell migration: a multiscale tuning model. J Cell Biol. 512 188(1), 11-9 (2010).

51313 Schor SL, Ellis IR, Harada K et al. A novel 'sandwich' assay for quantifying chemo514 regulated cell migration within 3-dimensional matrices: wound healing cytokines 515 exhibit distinct motogenic activities compared to the transmembrane assay. Cell Motil 516 Cytoskeleton. 63(5), 287-300 (2006).

51714 Lee EJ, Hwang CM, Baek DH, Lee SH. Fabrication of microfluidic system for the 518 assessment of cell migration on 3D micropatterned substrates. Conf Proc IEEE Eng 519 Med Biol Soc. 2009, 6034-6037 (2009).

52015 Zaman MH, Trapani LM, Sieminski AL et al. Migration of tumor cells in 3D 521 matrices is governed by matrix stiffness along with cell-matrix adhesion and 522 proteolysis. Proc Natl Acad Sci U S A. 103(29), 10889-94 (2006).

52316 Raghavan S, Shen CJ, Desai RA, Sniadecki NJ, Nelson CM, Chen CS. Decoupling 524 diffusional from dimensional control of signaling in 3D culture reveals a role for 525 myosin in tubulogenesis. J Cell Sci. 123(Pt 17), 2877-2883 (2010). 
52617 Lee SH, Miller JS, Moon JJ, West JL. Proteolytically degradable hydrogels with a

527 fluorogenic substrate for studies of cellular proteolytic activity and migration. 528 Biotechnol Prog. 21(6), 1736-1741 (2005).

52918 Sundararaghavan HG, Saunders RL, Hammer DA, Burdick JA. Fiber alignment 530 directs cell motility over chemotactic gradients. Biotechnol Bioeng. 110(4), 1249-1254 531 (2013).

53219 Raeber GP, Lutolf MP, Hubbell JA. Molecularly engineered PEG hydrogels: a novel 533 model system for proteolytically mediated cell migration. Biophys J. 89(2), 1374-1388 534 (2005).

53520 Hughes CS, Postovit LM, Lajoie GA. Matrigel: a complex protein mixture required 536 for optimal growth of cell culture. Proteomics. 10(9), 1886-1890 (2010).

53721 Hynes RO. Integrins: bidirectional, allosteric signaling machines. Cell. 110(6), 673$538687(2002)$.

53922 Burridge K, Chrzanowska-Wodnicka M. Focal adhesions, contractility, and 540 signaling. Annu Rev Cell Dev Biol. 12, 463-518 (1996).

54123 Schwartz MA, Ginsberg MH. Networks and crosstalk: integrin signalling spreads. $542 \quad$ Nat Cell Biol. 4(4), E65-8 (2002).

54324 Moghe PV, Ezzell RM, Toner M, Tompkins RG, Martin LY. Role of $\beta 1$ Integrin 544 Distribution in Morphology and Function of Collagen-Sandwiched Hepatocytes. Tissue 545 Engineering, 3(1), 1-16 (1997) 
25 Ballester-Beltrán J, Lebourg M, Rico P, Salmerón-Sánchez M. Dorsal and ventral stimuli in cell-material interactions: effect on cell morphology. Biointerphases. 7(1-4), 39 (2012).

26 Ballester-Beltrán J, Lebourg M, Salmerón-Sánchez M. Dorsal and ventral stimuli in sandwich-like microenvironments. Effect on cell differentiation. Biotechnol Bioeng. 110(11), 3048-3058 (2013).

27 Ballester-Beltrán J, Moratal D, Lebourg M, Salmerón-Sánchez M. Fibronectinmatrix sandwich-like microenvironments to manipulate cell fate. Biomater Sci. 2, 381389 (2014).

*Importance of sandwich culture conditions for cell behavior

28 Rasband WS. ImageJ U.S. National Institutes of Health, Bethesda, Maryland, USA, http://imagej.nih.gov/ij/1997-2012

29 Glaß M, Möller B, Zirkel A et al. Cell Migration Analysis: Segmenting Scratch Assay Images with Level Sets and Support Vector Machines. Pattern Recognition. 45(9), 3154-3165 (2012).

30 O’Connell B. Oval Profile Plot. Research Services Branch, National Institute of Mental Health, National Institute of Neurological Disorders and Stroke. Available from: http://rsbweb.nih.gov/ij/plugins/oval-profile.html. Accessed 20 March 2014.

31 Berginski ME, Gomez SM. The Focal Adhesion Analysis Server: a web tool for analyzing focal adhesion dynamics. F1000Research (2), 68 (2013).

http://faas.bme.unc.edu Accessed July 2014. 
56732 Kam Y, Guess C, Estrada L, Weidow B, Quaranta V. A novel circular invasion assay

568 mimics in vivo invasive behavior of cancer cell lines and distinguishes single-cell

569 motility in vitro. BMC Cancer. (8), 198 (2008).

57033 Lauffenburger DA, Horwitz AF. Cell migration: a physically integrated molecular 571 process. Cell. 84(3), 359-369 (1996).

57234 Ridley AJ, Schwartz MA, Burridge K et al. Cell migration: integrating signals from 573 front to back. Science. 302(5651), 1704-1709 (2003).

57435 Decaestecker C, Debeir O, Van Ham P, Kiss R. Can anti-migratory drugs be 575 screened in vitro? A review of 2D and 3D assays for the quantitative analysis of cell 576 migration. Med Res Rev. 27(2), 149-176 (2007).

57736 Fraley SI, Feng Y, Krishnamurthy R et al. A distinctive role for focal adhesion 578 proteins in three-dimensional cell motility. Nat Cell Biol. 12(6), 598-604 (2010).

57937 Nagano M, Hoshino D, Koshikawa N, Akizawa T, Seiki M. Turnover of focal 580 adhesions and cancer cell migration. Int J Cell Biol. 2012, 310616 (2012)

58138 Pankov R, Endo Y, Even-Ram S et al. A Rac switch regulates random versus 582 directionally persistent cell migration. J Cell Biol. 170(5), 793-802 (2005).

58339 Beningo KA, Dembo M, Wang YL. Responses of fibroblasts to anchorage of dorsal 584 extracellular matrix receptors. Proc Natl Acad Sci U S A. 101(52), 18024-18029 (2004).

$585 * *$ Cell behavior under polyactylamide sandwich cultures

58640 Hakkinen KM, Harunaga JS, Doyle AD, Yamada KM. Direct comparisons of the 587 morphology, migration, cell adhesions, and actin cytoskeleton of fibroblasts in four 
588 different three-dimensional extracellular matrices. Tissue Eng Part A. 17(5-6), 713-724

589 (2011).

$590 * *$ Deep study of cell migration in 3D systems

59141 Ochsner M, Textor M, Vogel V, Smith ML. Dimensionality controls cytoskeleton

592 assembly and metabolism of fibroblast cells in response to rigidity and shape. PLoS 593 One. 5(3), e9445 (2010).

59442 Harunaga JS, Yamada KM. Cell-matrix adhesions in 3D. Matrix Biol. 30(7-8), 363$595368(2011)$.

59643 Kim DH, Wirtz D. Focal adhesion size uniquely predicts cell migration. FASEB J. 597 27(4), 1351-1361 (2013).

59844 Collier IE, Wilhelm SM, Eisen AZ et al. H-ras oncogene-transformed human 599 bronchial epithelial cells (TBE-1) secrete a single metalloprotease capable of degrading 600 basement membrane collagen. J Biol Chem. 263(14), 6579-6587 (1988).

60145 Knäuper V, Cowell S, Smith B et al. The role of the C-terminal domain of human 602 collagenase-3 (MMP-13) in the activation of procollagenase-3, substrate specificity, and 603 tissue inhibitor of metalloproteinase interaction. J Biol Chem. 272(12), 7608-7616 604 (1997).

60546 Gugutkov D, Altankov G, Rodríguez Hernández JC, Monleón Pradas M, Salmerón 606 Sánchez M. Fibronectin activity on substrates with controlled --OH density. J Biomed 607 Mater Res A. 92(1), 322-31 (2010). 
47 Mao Y, Schwarzbauer JE. Stimulatory effects of a three-dimensional microenvironment on cell-mediated fibronectin fibrillogenesis. J Cell Sci. 118(Pt 19), 4427-4436 (2005).

611

48 Roca-Cusachs P, Iskratsch T, Sheetz MP. Finding the weakest link: exploring 612 integrin-mediated mechanical molecular pathways. J Cell Sci. 125(Pt 13), 3025-3038 (2012).

614

49 Doyle AD, Wang FW, Matsumoto K, Yamada KM. One-dimensional topography 615 underlies three-dimensional fibrillar cell migration. J Cell Biol. 184(4), 481-490 (2009).

61650 Narumiya S, Ishizaki T, Uehata M. Use and properties of ROCK-specific inhibitor 617 Y-27632. Methods Enzymol. 325:273-284 (2000).

51 Kovács M, Tóth J, Hetényi C, Málnási-Csizmadia A, Sellers JR. Mechanism of 619 blebbistatin inhibition of myosin II. J Biol Chem. 279(34):35557-35563 (2004).

52 Fitzpatrick FA, Wheeler R. The immunopharmacology of paclitaxel (Taxol), docetaxel (Taxotere), and related agents. Int Immunopharmacol. 3(13-14):1699-1714 622 (2003).

53 Ballester-Beltrán J, Rico P, Moratal D, Song W, Mano JF, Salmerón-Sánchez M. Role of superhydrophobicity in the biological activity of fibronectin at the cell-material interface. Soft Matter. (7), 10803-10811 (2011) fibronectin into networks: influence of surface chemistry and effect on osteoblast adhesion. Tissue Eng Part A. 15(11), 3271-3281 (2009). 
63055 Ballester-Beltrán J, Cantini M, Lebourg M et al. Effect of topological cues on 631 material-driven fibronectin fibrillogenesis and cell differentiation. J Mater Sci Mater 632 Med.23(1), 195-204 (2012).

63356 Salmerón-Sánchez M, Rico P, Moratal D, Lee TT, Schwarzbauer JE, García AJ. 634 Role of material-driven fibronectin fibrillogenesis in cell differentiation. Biomaterials. 635 32(8), 2099-2105 (2011).

63657 Llopis-Hernández V, Rico P, Moratal D, Altankov G, Salmerón-Sánchez M. Role of 637 material-driven fibronectin fibrillogenesis in protein remodeling. Biores Open Access. 638 2(5), 364-373(2013).

63958 Gonzalez-Garcia C, Cantini M, Ballester-Beltran J, Salmeron-Sanchez M. Nanoscale 640 mobility of fibronectin at the material interface determines cell fate. Submitted 